\title{
La educación empresarial en gestión de operaciones integrada al desarrollo de emprendimientos innovadores
}

\section{Business education in operational management integrated to the development of innovative enterprises \\ Edgar Vinicio Manzano Coello. ${ }^{1}$, \& Mario Patricio Padilla Martínez. ${ }^{2}$

\begin{abstract}
.
DOI: https://doi.org/10.33262/visionariodigital.v4i4.1417
\end{abstract}

Business education is understood as the improvement of the mental faculties of each individual, it should be noted that, to create, develop and innovate, in the first place, their professional preparation generates people with sufficient character of decision and objective determination, always based on technological and intellectual training, focusing on decision making that are necessary throughout working life. At present, humanity participates in a competitive environment, in which it is not only enough to finish a third level degree, but rather to opt for a postgraduate degree. Therefore, the student community is forced to continue with its academic training in universities in Ecuador, which have created postgraduate degrees for professionalization purposes in order to contribute to social, human and technological development. There are offers of studies with the necessary approaches to promote growth and personal evolution with approaches to operations management promoting the creation, development and organization of the production function in order to achieve competitive advantages. The purpose of the University is to develop the objective thinking of its students, thus meeting the needs of the national environment, structuring higher education, previously conducting a market analysis, which evaluates the significant growth of companies between 2012 and 2016 being of great importance the staff working in such companies and professionals trained to hold such positions covering such job competition. In this context, universities play an important role in creating fertile and knowledge-intensive environments to support the exploration and exploitation of innovative ideas and ventures, where governments have created grants to promote entrepreneurial innovation through mandatory university partnerships. Based on these ideas, the purpose of this documentary research is to analyze and analyze the role of universities

\footnotetext{
${ }^{1}$ Universidad Técnica de Ambato, Ambato, Ecuador, emanzano0966@uta.edu.ec.
}

${ }^{2}$ Universidad Técnica de Ambato, Ambato, Ecuador, mp.padilla@uta.edu.ec. 
in business education in operations management integrated with the development of new innovative ventures in relation to the quality of the professional.

Keywords: Enterprise, Entrepreneurship, Business Administration, Operations Management.

\section{Resumen.}

Se entiende como educación empresarial al perfeccionamiento de las facultades mentales de cada individuo, cabe indicar que, para crear, desarrollar e innovar, en primer lugar, su preparación profesional genera personas con el suficiente carácter de decisión y determinación objetiva, basándose siempre en la formación tecnológica e intelectual, enfocándolas en la toma de decisiones que son necesarias a través de la vida laboral.

En la actualidad la humanidad es partícipe de un ambiente competitivo, en el que no solo basta con terminar una carrera de tercer nivel sino más bien optar por un posgrado por ende la comunidad estudiantil se ve forzada a continuar con su capacitación académica en universidades del Ecuador mismas que han creado posgrados con fines de profesionalización a fin de contribuir al desarrollo social, humano y tecnológico.

Existen ofertas de estudios con los enfoques necesarios que impulsen el crecimiento y la evolución personal con enfoques a la gestión de operaciones impulsando la creación, desarrollo y organización de la función de producción con el objetivo de alcanzar ventajas competitivas. La Universidad tiene por finalidad de desarrollar el pensamiento objetivo de sus educandos logrando de esta manera satisfacer las necesidades del entorno nacional, estructurando la educación superior, realizándose previamente un análisis de mercado, donde se evalúa el crecimiento significativo de las empresas entre los años 2012 y 2016 siendo de gran importancia el personal que labora en dichas compañías y profesionales capacitados para ejercer puestos cubriendo dicha competencia laboral.

En este contexto, las universidades desempeñan un papel importante en la creación de entornos fértiles y de gran densidad de conocimientos para apoyar la exploración y explotación de ideas innovadoras y emprendimientos, donde los gobiernos han creado subvenciones para promover la innovación empresarial a través de asociaciones universitarias obligatorias. Basándose en estas ideas, el propósito de esta investigación documental es analizar el papel de las universidades en la educación empresarial en gestión de operaciones integrada al desarrollo de nuevos emprendimientos innovadores con relación a la calidad del profesional. 
Palabras claves: Empresa, Emprendimiento, Administración De Empresas, Gestión De Operaciones.

\section{Introducción.}

La educación empresarial se basa en modificar el pensamiento analítico del entorno en que vive cada individuo y que para afrontar los desafíos del futuro más próximo se debe mejorar los sistemas educativos realizando reformas curriculares en los niveles de estudio generando cambios en las estrategias pedagógicas en la formación de los profesionales del mañana (Marchesi \& Tedesco, 2019).

Mediante el cambio curricular desde las bases escolares la posibilidad de mejoramiento empresarial será mucho mayor, ya que se podría generar una cadena de valor poniendo en práctica la gestión de operaciones enfocadas a ideas nuevas de emprendimiento que aporte a la economía del país. Además, las iniciativas más exitosas se las están desarrollando a nivel universitario ya que se están implementando estudios empresariales fomentando el desarrollo de emprendimientos innovadores y a su vez volviendo realidad sus ideas de negocios.

Muchos estudios lo han discutido ampliamente. Lechner y Dowling (2003) estudiaron las redes egocéntricas de empresas emprendedoras de alto crecimiento en la industria y exploraron cómo estas empresas crecen a través del uso de las relaciones externas y se vuelven competitivas sobre la base de la investigación y cursos de posgrado realizado por sus universidades. Los resultados muestran que el crecimiento de las empresas emprendedoras depende no sólo de la construcción de redes egocéntricas, sino también de la existencia y el desarrollo de redes socio céntricas saludables (Ovalle, Olmeda, \& Perianes, 2016).

Zhang y Si (2008) exploran los factores externos clave que influyen en el rendimiento de las empresas emprendedoras de alta tecnología utilizando el método de encuesta por cuestionario, y los resultados muestran que una buena relación con una organización tiene un impacto positivo en el crecimiento de las empresas emprendedoras de alta tecnología y cursos de posgrado realizados en áreas específicas, sin embargo de una relación estrecha con el gobierno tiene un impacto negativo en el crecimiento de las empresas emprendedoras de alta tecnología. 
Dependiendo de los propósitos estratégicos, las empresas suelen colaborar con organizaciones de base científica, comercial y mixta (Alcalde \& Guerrero, 2016). En primer lugar, las empresas que se orientan hacia nuevas fuentes de empleo, entre ellas ideas innovadoras de emprendimiento que participan en la colaboración científica con las universidades (Kenney \& Mustar, 2014). En este sentido, los objetivos estratégicos de las asociaciones universidad-empresa son el aprendizaje/adquisición de nuevos conocimientos externos, la regeneración tecnológica, la absorción de nuevas habilidades y el desarrollo de emprendimientos a largo plazo, mediante, por ejemplo, la creación de redes o la subcontratación (Capon \& Farley, 2016).

En segundo lugar, las empresas orientadas a la generación de nuevos emprendimientos de manera incremental suelen participar en colaboraciones comerciales con diferentes socios vinculados a las filiales o a la cadena de valor industrial (salidas de conocimiento). Tradicionalmente, en lugar de prácticas de innovación basadas en la ciencia, las prácticas de innovación comercial se caracterizan por un rápido retorno de la inversión, la reducción del riesgo y la mejora de la flexibilidad, la calidad y la adaptabilidad del mercado (Dutrénit, 2017). En este sentido, estas prácticas también pueden perseguir objetivos no tecnológicos como la identificación de oportunidades y la expansión de los mercados de las empresas, mediante la internacionalización o la entrada en nuevos mercados (Puig, 2017). La evidencia sugiere alguna ventaja detrás de este tipo de prácticas de innovación, tales como la reducción de la incertidumbre del mercado, la concesión de licencias externas de propiedad intelectual, la capitalización de iniciativas y el retorno de la inversión (Marcet, 2015).

En tercer lugar, para lograr una exploración/explotación efectiva de una idea de emprendimiento, las empresas suelen adoptar patrones de colaboración diversificados. En este caso, el propósito es una combinación de alianzas que facilite la generación de emprendimientos nuevos (Alcalde \& Guerrero, 2016). En consecuencia, tener una variedad de socios comerciales y científicos tiene un mayor impacto positivo en el grado de novedad de los productos que la colaboración con un solo tipo de socio (Pavón, 2017), especialmente en contextos de prosperidad. En las economías emergentes, el acceso limitado a diversas fuentes financieras puede generar dinámicas empresariales negativas debido a la entrada insuficiente de empresas innovadoras en el mercado (García, 2018); por esta razón, las 
colaboraciones mixtas facilitan la creación de ventajas competitivas sostenibles y apoyan el rendimiento de la innovación (Henrekson, 2010), en particular para las empresas que luchan por la supervivencia organizativa, ya que las combinaciones óptimas de habilidades comerciales y técnicas (científicas) reducen la incertidumbre del mercado. Intuitivamente, en escenarios con condiciones de mercado impredecibles, las empresas intentarán colaborar con varios socios simultáneamente para ser competitivas e innovadoras, compartiendo al mismo tiempo los posibles retos y riesgos.

El emprendimiento no solo atrae beneficios económicos a nivel del país por disponer de personas aptas para generar sus propias ocupaciones, sino que también aporta al desarrollo individual de los jóvenes, fomentando su autoestima y confianza (Olmos, 2012). Los estudiantes universitarios aprenden actitudes, habilidades, comportamientos que en situaciones especiales les permitirán entrar en el mundo del trabajo y continuar con su carrera profesional con ingresos económicos extras (Duarte, 2017).

La gestión de operaciones integrada al emprendimiento empresarial como base del desarrollo y crecimiento del país a traer consigo ideas de nuevas empresas productivas que aportan a la sociedad, sin embargo, a pesar de que instituciones universitarias sirven de guía y brindan herramientas para una gestión de operaciones efectiva, es limitado el uso de nuevas ideas para generar ideas emprendedoras (Oyarvide, 2016).

La investigación se justifica pues es indiscutible que el aprendizaje actualizado del emprendimiento y la gestión de operaciones contribuye en forma positiva el incremento de las posibilidades laborales en el área empresarial. Además, es viable ya que cuenta con acceso a información bibliográfica comprobada que permitirá desarrollar una investigación de manera adecuada, comparándola con fuentes académicas y científicas, el levantamiento de la información será debidamente revisada y validada en fuentes estadísticas avaladas por las entidades educativas pertenecientes a la provincia de Tungurahua, además de tomar en consideración investigaciones anteriores con el fin de corroborar los respectivos datos. De esta manera la investigación pretende caracterizar la influencia de la educación empresarial que brindan entidades educativas en gestión de operaciones asociándolo con perfiles de competencias que son parte de la formación empresarial de los estudiantes, donde se destacan variables a considerar como el género, edad, años de experiencia, fuentes de financiamiento 
y de conocimiento. Además, aspectos externos a la educación como necesidad de superación con la generación de nuevas ideas innovadoras de emprendimientos en base a la gestión de operaciones. De esta manera se plantean los siguientes objetivos.

- Analizar el papel de las universidades en la educación empresarial en gestión de operaciones integrada al desarrollo de nuevos emprendimientos innovadores con relación a la calidad del profesional.

- Identificar el perfil de los egresados analizado en la literatura relacionándolo con la educación empresarial y gestión de operaciones.

- Determinar el nivel ocupacional de los egresados que han generado ideas de emprendimiento después de sus estudios en educación empresarial en gestión de operaciones.

\section{Marco teórico.}

\section{Empresa.}

Se considera que una empresa es cualquier entidad que ejerza una actividad económica, con independencia de su forma jurídica. Esto incluye, en particular, a los trabajadores por cuenta propia y a las empresas familiares que realizan actividades artesanales o de otro tipo, así como a las asociaciones que ejercen regularmente una actividad económica, cuyo objetivo es la consecución de un beneficio a través de la satisfacción de una necesidad de mercado (Castillo, 2016). La satisfacción de las necesidades que plantea el mercado se concreta en el ofrecimiento de productos (empresa agrícola o sector primario, industrial o sector secundario, servicios o sector terciario), con la contraprestación de un precio (Rosa \& Arregoces, 2015).

\section{Emprendimiento.}

El emprendimiento implica el descubrimiento, evaluación y explotación de oportunidades, es decir, nuevos productos, servicios o procesos de producción; nuevas estrategias y formas de organización y nuevos mercados para productos e insumos que antes no existían (Rivera \& Israel, 2013). El emprendimiento es una oportunidad económica inesperada y aún no valorada. Las oportunidades empresariales existen porque los diferentes agentes tienen ideas diferentes sobre el valor relativo de los recursos o cuando los recursos se convierten de 
insumos en productos. La teoría del emprendedor se centra en la heterogeneidad de creencias sobre el valor de los recursos (Camelo, 2015). El espíritu empresarial puede conceptualizarse como el descubrimiento de oportunidades y la posterior creación de nueva actividad económica, a menudo a través de la creación de una nueva organización (Arriagada, 2002)

\section{Gestión de operaciones.}

Históricamente, todo comenzó con la división de la producción, comenzando ya en los tiempos de los antiguos artesanos, pero extendiéndose más ampliamente sólo añadiendo el concepto de intercambiabilidad de las piezas en el siglo XVIII, desencadenando finalmente la revolución industrial. A medida que las economías del mundo desarrollado cambiaban gradualmente para estar basadas en los servicios, todas las funciones corporativas, incluida la gestión de productos, comenzaron a integrarlas. El lado de los servicios también comenzó su enfoque aplicando los principios de gestión de productos a la planificación y organización de los procesos, hasta el punto de que tenía más sentido llamarlo gestión de operaciones (Garces, 2016).

La gestión de operaciones implica la planificación, organización y supervisión de los procesos, así como la introducción de las mejoras necesarias para lograr una mayor rentabilidad (Castillo, 2016). Los ajustes en las operaciones diarias tienen que apoyar los objetivos estratégicos de la empresa, por lo que van precedidos de un profundo análisis y medición de los procesos actuales. Anteriormente, la gestión de operaciones se denominaba gestión de la producción, lo que demuestra claramente su origen en la fabricación.

\section{Habilidades requeridas en gestión de procesos.}

Según Arriagada (2002) las habilidades necesarias para realizar este tipo de trabajo son tan diversas como la propia función. Las habilidades más importantes son:

Habilidades organizativas. La organización de procesos en una organización requiere un conjunto de habilidades que van desde la planificación y priorización, pasando por la ejecución, hasta el monitoreo. Estas habilidades juntas ayudan al gerente a lograr productividad y eficiencia. 
Capacidades analíticas/comprensión del proceso. La capacidad de entender los procesos en su área a menudo incluye también una amplia comprensión de otras funciones. Una atención a los detalles es a menudo útil para profundizar en el análisis.

Coordinación de procesos. Una vez que los procesos son analizados y entendidos, pueden ser optimizados para una máxima eficiencia. La rapidez en la toma de decisiones es una ventaja real en este caso, así como una clara orientación para la resolución de problemas.

Habilidades con la gente. Las fallas en las interacciones con los empleados o miembros de la alta gerencia pueden perjudicar seriamente la productividad, por lo que un gerente de operaciones debe tener las habilidades de las personas para navegar adecuadamente las líneas finas con sus colegas. Además, la comunicación clara de las tareas y objetivos sirve como gran motivación y para dar un propósito para todos.

Creatividad. Una vez más, las habilidades para resolver problemas son esenciales para un enfoque creativo si las cosas no van en la dirección correcta. Cuando lo hacen, la creatividad ayuda a encontrar nuevas formas de mejorar el rendimiento corporativo.

Ahorro en tecnología. Para entender y diseñar procesos en una época en la que las operaciones son cada vez más dependientes de la tecnología, la afinidad por la tecnología es una habilidad que no se puede subestimar. Los gerentes de operaciones deben estar familiarizados con las tecnologías más comunes utilizadas en sus industrias y tener un conocimiento aún más profundo de la tecnología de operaciones específica de sus organizaciones.

Según Aguilar (2017), menciona que el perfil de los trabajadores y emprendedores de los tungurahuenses vienen marcados por ciertos factores que se exponen a continuación.

Las características personales contemplan variables como la creatividad, donde se menciona que las personas sienten constantemente el impulso de crear ideas originales o diferentes, además consideran poseer un pensamiento flexible y capacidad imaginativa, poseen habilidad para resolver las dificultades que se le presentan a través de la generación de nuevas ideas. 
En cuanto a la toma de decisiones como parte del perfil emprendedor un $78 \%$ de la muestra de estudio a la hora de tomar decisiones seleccionan las situaciones más relevantes a atender; el $85 \%$ de las personas encuestadas buscan posibles alternativas de solución y se informa detalladamente frente a situaciones que se presentan. Además, el $90 \%$ de los entrevistados menciona que la búsqueda de la solución la realiza teniendo en cuenta los recursos que posee y está en capacidad de adquirir.

Por otra parte, respecto a la responsabilidad un 75\% normalmente identifica las consecuencias que pueden tener sus acciones sobre él y otras personas, mientras que un $87 \%$ considera que una empresa debe velar no solo por su crecimiento económico, sino que además debe trabajar para aportar al equilibrio social y ambiental, un 90\%piensa que cuando se inicia una idea de emprendimiento, se adquiere obligaciones tanto legales como éticas.

\section{Materiales y métodos.}

La educación empresarial y la gestión de operaciones debe ser racionalizada, pero requiere de la personalización y el compromiso de los líderes de la organización. Para generar conocimiento sobre cómo implementar el concepto de gestión de la innovación, se recurre a un enfoque de investigación-acción. Voss y otros (2002) mencionan que la experimentación es necesaria para este tipo de investigación y mencionan que los estudios de caso son un método de investigación adecuado. La misma puede verse como una variación de la investigación de casos, en la que el investigador de acción no es un observador independiente. Esta investigación se basa en un estudio de investigación-acción que pretende explorar los elementos y procesos que permiten a un estudiante pos graduación implantar el concepto de gestión de operaciones.

La investigación documental cubre un período entre los años 2012-2016. Además, se realizará un análisis de los diversos factores empresariales que inciden la toma de decisiones al momento de realizar emprendimientos luego de egresar de la universidad, se realizarán modelaciones y encuestas que determinan la motivación o no de realizar emprendimientos o líneas de negocios ya sea de bienes o servicios.

La recolección de datos parte de un estudio netamente bibliográfico, pues se toma a consideración reportes e informes de seguimientos a graduados de la facultad de ciencias 
administrativas de los últimos años, la misma se ha realizado con una muestra calculada de la totalidad de graduados en los años de estudio. Los resultados son obtenidos mediante encuestas en base a cuestionarios estructurados con preguntas cerradas que son plasmados en investigaciones anteriores. De esta manera se compara con previos análisis tanto de docentes como de graduados en sus artículos y proyectos de investigación, los mismos que forman parte de la realidad y problemática de este documento.

\section{Resultados.}

Tomando como referencia al sistema de seguimiento a graduados, se procede a levantar la información de los graduados, con el fin de medir su avance académico y la evolución ocupacional.

Tabla 1. Variables de análisis

\begin{tabular}{ccccc}
\hline \multicolumn{2}{c}{ Genero } & \multicolumn{3}{c}{ Año de graduación } \\
\hline Masculino & Femenino & $\mathbf{2 0 1 2 - 2 0 1 4}$ & $\mathbf{2 0 1 4 - 2 0 1 6}$ & $\mathbf{2 0 1 6 - 2 0 1 8}$ \\
$22 \%$ & $78 \%$ & $25 \%$ & $41 \%$ & $33 \%$ \\
\hline
\end{tabular}

Fuente: (Informe de seguimiento a graduados, 2017)

Elaborado por: Manzano y Padilla, 2019.

Tabla 2. Condición laboral vs cargo desempeñado después de posgrado

Condición laboral

\section{Cargo desempeñado después de posgrado}

\begin{tabular}{cccc}
\hline Autónomo & $7 \%$ & Dto. ventas & $35 \%$ \\
Sector Privado & $71 \%$ & Dto. Distribución & $34 \%$ \\
Sector público & $15 \%$ & Dto. Contable & $26 \%$ \\
Otros & $6 \%$ & Dto. Talento Humano & $5 \%$ \\
\hline
\end{tabular}

Fuente: (Informe de seguimiento a graduados, 2017)

Elaborado por: Manzano y Padilla, 2019

La tabla 1 indica variables de investigación como género, año de graduación, condición laboral después de su posgrado. Los resultados apuntan a una mayoría de personas del sexo femenino en los últimos años, siendo este un $78 \%$, frente a un $22 \%$ de masculino. Además, 
en cuestión al año de graduación entre los años 2012-2016 existe un porcentaje elevado en relación a años anteriores y posteriores.

En la tabla 2 indica que, en cuestión al desarrollo de la condición laboral, la mayoría de egresados, representativos de un $71 \%$ forman parte del sector privado, cabe recalcar que es en esta sección donde se encuentra la mayor cantidad de estudiantes, donde se aplica de cierto modo la gestión empresarial. Por otro lado, las condiciones laborales autónomas, hacen referencia a emprendimientos propios solo forma parte de un 6\% de los egresados de posgrados de la Universidad Técnica de Ambato, dicho valor indica que a pesar de los estudios realizados en cursos posteriores al grado de los estudiantes no se han generado trabajos autónomos con enfoques y miras de emprendimiento. Por otra parte, los valores de cargos que destacan en el lugar de trabajo de dichas personas oscilan entre departamento de ventas, distribución y contable con porcentajes de 35\%, 34\% y $26 \%$ respectivamente.

\section{Causas y razones por la cual no se realice un emprendimiento propio}

Tabla 3. Limitaciones de un emprendimiento

\begin{tabular}{lclc}
\hline Causas & Porcentaje & Razones & Porcentaje \\
\hline Estabilidad laboral & $18,18 \%$ & Falta de recursos & $47,33 \%$ \\
Beneficios Sociales & $18,18 \%$ & Falta de ideas de negocio & $29,33 \%$ \\
Miedo al fracaso & $18,18 \%$ & Falta de tiempo & $23,33 \%$ \\
Falta de financiamiento & $45,45 \%$ & Otros & $0,00 \%$ \\
Total & $100 \%$ & Total & $100 \%$ \\
\hline
\end{tabular}

Fuente: (González \& Vera, 2015)

Elaborado por: Manzano y Padilla, 2019

Las causas de porque siguen laborando en relación de dependencia y no realizan emprendimiento, el 45,45\% indican que la falta de financiamiento es una causa principal, seguido de un $18 \%$ que establece diferentes causas entre ellas estabilidad laboral, beneficios sociales y miedo al fracaso. A todo esto, se puede determinar que existen varios factores entre ellos la falta de dinero para que los encuestados no realicen emprendimiento propio. Por otra parte, Del total de encuestados el 47,33\% indicó que por falta de recursos es que no han iniciado un negocio, el 29,33\% manifestó que la falta de ideas de negocio es otro aspecto por 
el cual no han empezado a emprender, y el 23,33\% señaló que la falta de tiempo es una de las razones. Entonces se puede determinar que los egresados no poseen recursos necesarios para iniciar un negocio puesto que muchos de ellos laboran en una empresa en la cual mantienen una dependencia laboral.

\section{Discusión.}

Según los resultados de la investigación bibliográfica y documental, tanto el género masculino como el género femenino en su mayoría, se encuentran trabajando, sin embargo, quienes no han conseguido un empleo puede deberse a los índices de desempleo que se enfrentan o a su vez a la alta competencia laboral que existe por las Áreas Administrativas. La condición actual de los graduados encuestados, denota que el porcentaje de personas que se encuentran trabajando en el sector privado sigue siendo mayor que el resto de empresas, sin embargo, en comparación a su condición cuando recién se graduaron ha disminuido, esto debido a que otros encontraron mejores opciones y accedieron al sector público, así como a otras personas se les acabaron sus contratos y están hoy en día desempleados por lo que optan muchos de ellos emprender. Es así que, debido a las mejores oportunidades en el campo laboral y personal que la constante preparación académica aporta, el interés por estudios de cuarto nivel, es similar tanto en el género masculino como femenino.

Así, los directivos consideran que la eficacia del enfoque del marco de gestión de operaciones era demostrable para todos los interesados, ya que ayudaba a estructurar el concepto de gestión de operaciones y conseguía integrarlo en las actividades cotidianas de la empresa. No obstante, no todos los elementos del marco propuesto se utilizaron en la misma medida ni fueron evaluados por igual por los administradores.

Por lo tanto, a la hora de iniciar el proceso de implantación un nuevo emprendimiento, hay que tener en cuenta que no todas las áreas del marco de gestión de operaciones tienen que desplegarse en la misma medida, sino los objetivos y la estrategia específicos de la empresa, así como el enfoque de los directivos.

La gestión de operaciones se enfoca en la ejecución de todas aquellas acciones tendientes a presentar el mayor valor agregado mediante la planificación, organización, dirección y control en la distribución de un producto o servicio en una empresa. A nivel estratégico, los 
estudiantes egresados de posgrados en gestión de operaciones y educación empresarial en general poseen dichas habilidades y funciones, sin embargo, en la realidad son factores externos a la educación lo que cual genera el rechazo por la creación de emprendimientos propios con ideas innovadoras. En un mundo tan globalizado donde todo apunta a aumentar la calidad, productividad, mejorar la satisfacción de los clientes y disminuir en lo mínimo los costes es necesario participar en la búsqueda de estrategias que sirvan de ventaja competitiva y sustentable para las futuras organizaciones, y estas deben estar plasmadas en la educación empresarial.

\section{Conclusiones.}

- El propósito de esta investigación bibliográfica y documental fue proporcionar una mejor comprensión del papel de las universidades en el desarrollo de nuevos emprendimientos de graduados en las economías emergentes. Para lograr este objetivo, en el contexto de la universidad, se analizaron variables que limitan el desarrollo de una idea de emprendimiento, en donde el financiamiento fue uno de los factores que más resaltan según investigaciones anteriores.

- Si se desea adquirir una educación empresarial que genere impacto y deseo de crear nuevos emprendimientos con visión innovadora, y aportar a tener un tejido empresarial de calidad que genere desarrollo económico, las universidades tanto públicas y privadas deben comprometerse más con la educación empresarial y el enfoque en la gestión de operaciones. Es así que, en el seno de la universidad se deben fortalecer los campos investigativos enfocados a la administración y la gestión de operaciones, fomentando la creación de grupos de centros y de institutos de investigación para la generación de nuevas ideas.

- De la población analizada, el porcentaje es mínimo de aquellos que cuentan con un empleo autónomo. Por otra parte, del total analizado un $71 \%$ forman parte del sector privado, cabe recalcar que es en esta sección donde se encuentra la mayor cantidad de estudiantes, donde se aplica de cierto modo la gestión empresarial, dicho valor indica que a pesar de los estudios realizados en cursos posteriores al grado de los estudiantes no se han generado trabajos autónomos con enfoques y miras de emprendimiento. 
- Los principales resultados de la investigación sugieren que, en primer lugar, se necesita una visión estratégica clara y compartida de los objetivos y retos que, centrándose en los procesos y proyectos empresariales clave, y combinando las herramientas y técnicas de gestión de la innovación existentes, se puede llevar a cabo un proceso de implementación de la gestión de la innovación con efectos positivos en la organización y en la calidad y aceptabilidad percibidas.

\section{Referencias bibliográficas.}

Aguilar, D. (2017). Factores determinantes del emprendimiento y la creación de nuevas empresas el caso de Tungurahua en el Ecuador. Abato: Universidad técnica de Ambato.

Alcalde, H., \& Guerrero, M. (2016). Open business models in entrepreneurial stages: Evidence from young Spanish firms during expansionary and recessionary periods. International Entrepreneurship and Management Journal, 393-413.

Arriagada, R. (2002). Diseño de un sistema de medición de desempelo para evaluar la gestión municipal: una propuesta metodológica. Santiago de Chile: Cepal.

Camelo, L. (2015). Elaboración de un plan de negocio como estrategia de desarrollo personal. Bogota: Universidad de la Salle.

Capon, N., \& Farley, J. (2016). Determinants of financial performance: A meta-analysis. Management Science, 1143-1159.

Castillo, C. (2016). Espíritu empresarial como estrategia de competitividad y desarrollo económico. Revista Escuela de Administración de Negocios, 103-117.

Dutrénit, G. (2017). La estrategia de innovación de la OCDE. Madrid.

Garces, M. (2016). Teoría Clásica del Comercio Internacional. Madrid: Universidad de Madrid.

García, C. (2018). Emprender en economías emergentes: el entorno institucional y su desarrollo. Emprendimiento y gestión empresarial, 133-155.

González, F., \& Vera, R. (2015). Análisis delas causas que limitan la realización de emprendimientos en los egresados de la escuela de ingeniería comercial. Guayaquil: Universidad Estatal de Guayaquil. 
Henrekson, M. (2010). The interaction of entrepreneur ship and institutions. Stockholm: Research Institute of Industrial Economic.

Kenney, M., \& Mustar, P. (2014). Entrepreneurial innovation: The importance of context. Research Policy, 1097-1108.

Lechner, C., \& Dowling, M. (2003). Firm networks: External relationships as sources for the growth and competitiveness of entrepreneurial firms. Entrepreneurship \& Regional Development: An International Journal, 1-26.

Marcet, X. (2015). La innovación es algo constante, necesita cierta gimnasia. Madrid: Universidad de Madrid.

Marchesi, A., \& Tedesco, J. (2019). Calidad, equidad y reformas en la enseñanza. Madrid: Fundacion Santillana.

Ovalle, A., Olmeda, C., \& Perianes, A. (2016). Una aproximación al análisis de Redes egocéntricas de colaboración interinstitucional. Revista hispana para el análisis de redes sociales, 11-18.

Palatchim, S. (2012). Revisión metodologica en las áreas de planeación y organizacipon aplicadas a PYMEs en la industria de la construcción. México: Universidad Iberoamericana.

Pavón, M. (2017). Gestión e innovación: un enfoque estratégico. Madrid: Pirámide.

Puig, G. (2017). Estrategias de innovación en las empresas de biotecnología catalanas. Barcelona: Universidad politécnica de Catalunya.

Rivera, G., \& Israel, S. (2013). Modelo de gestión para las empresas familiares con perspectivas de crecimiento y sostenibilidad. Perspectivas, 87-132.

Rosa, S., \& Arregoces, A. (2015). Diseño de un plan de mejoramiento administrativo para los departamentos de compra, logistuca y recursos humanos, de la empresa Buszca S.A.; en la ciudad de cartagena. Cartagena: Universidad de Cartagena.

Universidad técnica de Ambato. (2017). Informe de seguimiento a graduados. Ambato: Universidad Técnica de Ambato.

Zhang, Y., \& Si, C. (2008). The impacts of external factors on the growth of chinese entrepreneurial enterprises: An empirical study. Journal of Small Business \& Enterprise Development, 689-703. 


\section{PARA CITAR EL ARTÍCULO INDEXADO.}

Manzano Coello, E. V., \& Padilla Martínez, M. P. (2020). La educación empresarial en gestión de operaciones integrada al desarrollo de emprendimientos innovadores. Visionario Digital, 4(4), 32-47. https://doi.org/10.33262/visionariodigital.v4i4.1417

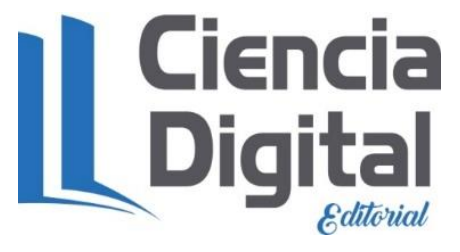

El artículo que se publica es de exclusiva responsabilidad de los autores y no necesariamente reflejan el pensamiento de la Revista Visionario Digital.

El artículo queda en propiedad de la revista y, por tanto, su publicación parcial y/o total en otro medio tiene que ser autorizado por el director de la Revista Visionario Digital.
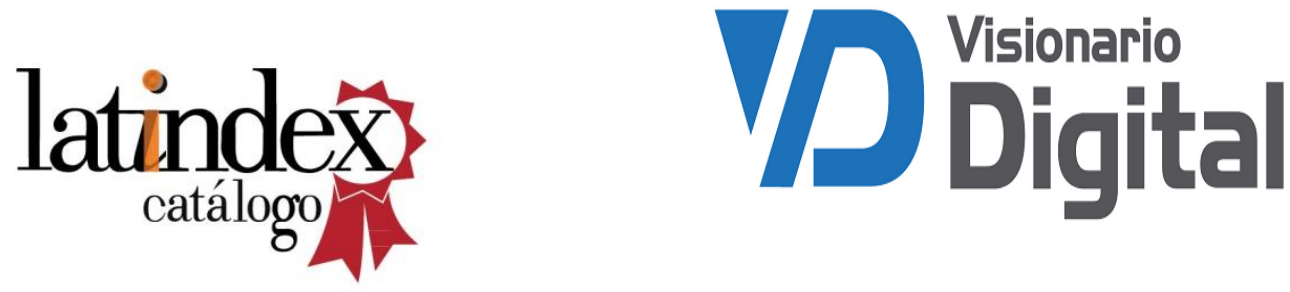